\title{
Are lice good proxies for host history? A comparative analysis of the Australian magpie, Gymnorhina tibicen, and two species of feather louse
}

\author{
A Toon and JM Hughes \\ School of Environment, Griffith University, Nathan, Queensland, Australia
}

Parasites have been recently advocated as useful proxies for unravelling a complex evolutionary history of a host. In the present study, two species of feather lice, Brueelia semiannulata and Philopterus sp. were analysed for mitochondrial variation and compared to mitochondrial and nuclear variation in their host, the Australian magpie Gymnorhina tibicen. Phylogenies constructed using maximum likelihood methods revealed geographic structuring for both species of feather lice and host. Our genetic analysis shows concordance of eastwest structure between host and Philopterus sp. indicating that it is an informative proxy for host history. Analysis of the
Philopterus sp. phylogeny also suggested cryptic structuring within the eastern magpie population that had not been previously realized through genetic analysis of the host. $B$. semiannulata however, did not show congruent phylogeographic structuring with the host. Rather than showing an eastwest split between lineages, the phylogeny of $B$. semiannulata showed north-south geographic structuring. It is postulated that this incongruence may be due to ecological habitat differences and/or the dispersal ability of $B$. semiannulata. Heredity (2008) 101, 127-135; doi:10.1038/hdy.2008.37; published online 7 May 2008

Keywords: Phthiraptera; avian phylogeography; feather parasites; mtDNA

\section{Introduction}

Comparative analysis of genetic variation among hostparasite systems provides insights into the ecology and evolutionary history of both the parasite and the host. Recently, several studies have demonstrated the usefulness of parasites as proxies for inferring history or ecology of the host (Nieberding and Olivieri, 2007). For example, genetic variation in parasites has been useful in determining recent history and demography in a threatened species of hawk (Whiteman et al., 2007). These studies comparing host and parasite phylogenies have revealed a number of patterns. Where there is high specificity of the parasite, molecular data may show congruent patterns with the host (Whiteman et al., 2006) and in some cases reveal cryptic structure in the host (Nieberding et al., 2004). A close association between parasite and host may lead to host races, or speciation (McCoy et al., 2005). In such cases, parasites may be useful in estimating dispersal and identifying source populations of the host (Criscione et al., 2006, Criscione and Blouin, 2007). In other cases, the genetic structure of parasite populations may be less than that of the host (Dybdahl and Lively, 1996), indicating higher dispersal of the parasite and/or the importance of ecological habitat differences (Nadler, 1995).

Correspondence: $\operatorname{Dr}$ A Toon, Integrative Biology, Brigham Young University, 401 WIDB, Provo, UT 84602-5181, USA.

E-mail: a_toon@byu.edu

Received 5 July 2007; revised 27 March 2008; accepted 28 March 2008; published online 7 May 2008
Various features of feather lice make them especially suitable for inferring history or ecology of their host. Many species of louse spend their entire life cycle on the host and disperse via direct contact between hosts (Marshall, 1981), suggesting a shared history among some species of lice and their hosts. In addition, parasitic lice have been shown to have an elevated rate of nearly neutral molecular evolution compared to their host (Page et al., 1998). On the basis of the idea of a shared evolutionary history with the host; this elevated rate of evolution may be utilized to uncover the recent history of the host.

The Australian magpie, Gymnorhina tibicen (Artamidae) is a widespread passerine endemic to Australia and Papua New Guinea. Three main plumage forms based on dorsal colour are currently recognized in Australia: black backed (BB) in northern Australia, white backed (WB) in southeastern Australia and Tasmania and white back males/black back females (varied) in southwestern Australia (Schodde and Mason, 1999). Phylogeographic analysis of magpie populations based on mitochondrial control region variation indicated shallow east-west structuring (Toon et al., 2007). It was estimated that populations had been isolated on either side of the continent in the late Pleistocene (less than 50000 years). Microsatellite analysis on the same populations suggested that in fact there had been secondary contact between northeastern and northwestern populations. It was suggested that male-mediated dispersal in magpies could account for the evidence of gene flow in nuclear genes preceding gene flow in mtDNA. 
Two species of chewing lice: Philopterus sp. (Nitzch, 1818; Ischnocera: Philopteridae) and Brueelia semiannulata (Piaget, 1883; Ischnocera: Philopteridae) are obligate ectoparasites of the Australian magpie. Philopterus sp. collected from mainland populations of G. tibicen belong to a single undescribed species (Hughes, 1984a). B. semiannulata is only known to parasitize the Australian magpie (Price et al., 2003). They are distantly related species and act as independent taxa for evolutionary questions. Louse morphology is not associated with the east-west mtDNA divergence (or back colour differences) among magpie host populations for either species of louse. In a morphometric analysis based on head characters, Hughes (1984b) reported little difference among mainland populations of $B$. semiannulata. Morphological variation was found between some mainland populations of Philopterus sp., although no consistent geographical pattern (for example, isolation by distance) was evident.

Philopterus sp. are highly habitat specific and restricted to the head and nape feathers of the magpie. In contrast, the habitat of $B$. semiannulata is much larger (body feathers) and includes a range of feather sizes, suggesting that it is a less specific species (Clay, 1951). Higher habitat specificity and lower habitat availability for Philopterus sp. may result in lower dispersal potential than for $B$. semiannulata due to less opportunities for Philopterus sp. to gain access to a new host through host to host contact. Some species of lice that share a host but live in different habitats also display differences in dispersal potential (Johnson et al., 2002). The differences in dispersal potential among the two louse species are expected to result in greater levels of gene flow among populations of $B$. semiannulata and possibly higher concordance of genetic variation with the host in Philopterus sp.

In the current study, genetic variation in two species of obligate feather lice is analysed and compared to the avian host to test the idea that parasites are valuable tools for reconstructing the evolutionary history of the host. Primarily, we were interested in testing the concordance of feather lice with the host. If structure is concordant, we asked whether additional information could be gleaned from using phylogenies of Philopterus sp. and B. semiannulata to infer host history? If structure is not concordant, then what are the non-historic factors shaping the population structure of the feather lice?

\section{Methods}

\section{Sampling and DNA extraction}

Lice were collected during a phylogeographic study on the host. Sites were targeted that represented the three main back colour forms of the Australian magpie. No Philopterus sp. samples were collected from southwestern magpie populations (varied magpie), even though more than 30 birds were captured and examined during sampling. Sampling localities are shown in Figure 1. Magpies were caught using an open-ended chicken wire cage placed over a caged live decoy magpie. Each wild bird was examined visually for the presence of Philopterus sp. and B. semiannulata by searching the feathers for 5-10 min. All lice were removed from the feathers of the host with feather-light forceps and preserved in $95 \%$

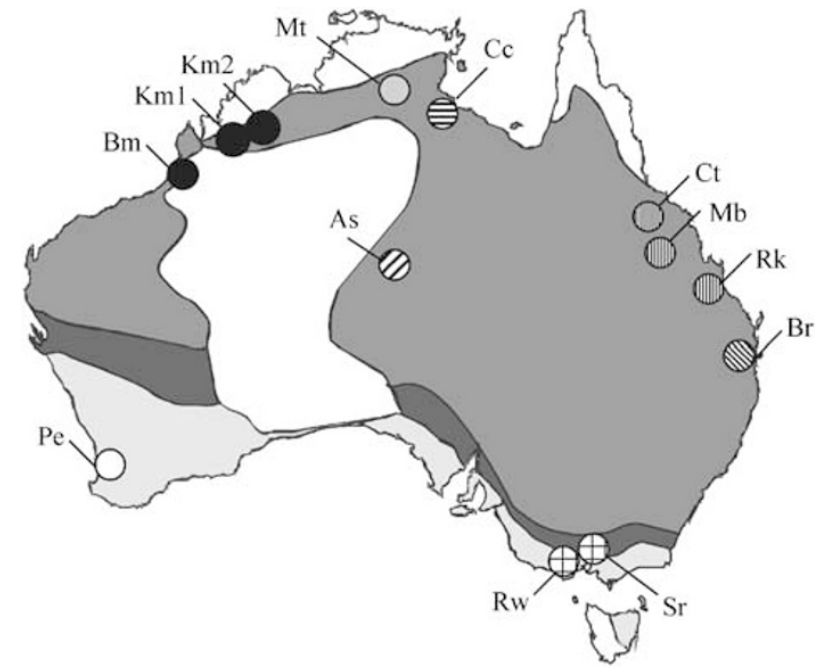

Figure 1 Sampling sites of G. tibicen, Philopterus sp. and $B$. semiannulata where each population is represented by a pattern that corresponds to haplotype networks in Figure 4. Australian magpie plumage forms adapted from Schodde and Mason (1999). Black-backed forms (BB) are highlighted in dark grey. White-backed forms (WB) are highlighted in light grey. Zones of inter-gradation between plumage forms are highlighted in black.

ethanol. A sample of blood was collected from the toenail of each magpie caught and stored in lysis buffer until samples were returned to the laboratory and stored at $-80{ }^{\circ} \mathrm{C}$.

Prior to extraction, lice were soaked in lysis buffer (0.02 M Tris- $\mathrm{HCl}, 0.05 \mathrm{M}$ EDTA (pH 8.0), $0.05 \mathrm{M} \mathrm{NaCl}$, $0.2 \%$ SDS) for $24 \mathrm{~h}$. A single louse was extracted at a time with the DNeasy Tissue Kit (Qiagen, Victoria, Australia) following the specified protocol for insects.

\section{Amplification and sequencing}

A fragment of cytochrome oxidase I (COI) was amplified and sequenced with the universal primers LCO1490 (5'GGTCAACAAATCATAAAGATATTGG-3') and HCO2198 (5'-TAAACTTCAGGGTGACCAAAAAATCA-3' (Folmer et al., 1994) for both species of feather lice. For the host, control region sequence variation was previously described in Baker et al. (2000) and Toon et al. (2007). Although use of an identical gene fragment for lice would have been preferred, the non-coding region of the mtDNA in lice is AT rich and greatly reduced in length (Covacin et al., 2006). In contrast, COI has been shown to be a highly variable marker that is useful for intraspecific studies of invertebrate species (Heilveil and Berlocher, 2006; Whiteman et al., 2007).

The target fragment was amplified in $25 \mu \mathrm{l}$ reactions containing $1 \times$ Taq polymerase buffer, $2 \mathrm{mM} \mathrm{MgCl}_{2}$, $0.2 \mathrm{mM}$ deoxyribonucleotide triphosphates, $0.4 \mu \mathrm{M}$ of each primer, 0.1 U Biotaq Taq polymerase (Bioline, New South Wales, Australia). The following programme was used to amplify the target fragment, after an initial denaturation of $5 \mathrm{~min}, 40$ cycles were completed of $95^{\circ} \mathrm{C}$ for $30 \mathrm{~s}, 50^{\circ} \mathrm{C}$ for $30 \mathrm{~s}, 72^{\circ} \mathrm{C}$ for $30 \mathrm{~s}$ followed by a final extension step of $72{ }^{\circ} \mathrm{C}$ for $5 \mathrm{~min}$. The PCR products were gel purified with Qiaquick PCR purification (Qiagen). The COI fragment was sequenced in both forward and reverse directions using an ABI377 automated sequencer 
(Griffith University Sequencing facility) following the standard big dye (ABI) sequencing protocol.

\section{Phylogenetics and statistical analysis}

Sequences were aligned with Sequencher 4.1 (Gene Codes Corporation). To help rule out the possibility that genetic patterns in the louse phylogeny were due to sequencing nuclear mitochondrial pseudogenes (Numts), sequences were visually inspected for the occurrence of stop codons and frameshift changes following Bensasson et al. (2001).

Phylogenetic analyses were completed in Paup version 4.0b10 (Swofford, 2000). An appropriate substitution model was selected with the use of Modeltest version 3.7 (Posada and Crandall, 1998). A Philopterus sp. sequence collected from the sister species (Cracticus nigrogularis) of the Australian magpie was used to root the Philopterus sp. data set. No outgroup was available for the $B$. semiannulata data set and thus the phylogeny was midpoint rooted. Mean divergence among haplotypes within clades and net mean divergence between clades (Nei and Li, 1979) was calculated using the selected substitution model. Maximum likelihood trees were constructed and 100 bootstrap replicates were performed on each of the aligned louse sequence data sets.

To compare host and louse phylogeographic structure, phylogenetic relationships among haplotypes were reconstructed with parsimony network analysis using TCS 1.21 (Clement et al., 2000) with a 95\% connection limit. Networks were constructed for each of the three mtDNA data sets. For the host, only populations where either species of louse was collected were included in the analysis. Due to the deep divergences among some louse sequences, we were unable to connect louse clades using TCS. Nonetheless, the use of a network allows the comparison of structure within clades among data sets. In addition, the ancestry among extant haplotypes can be identified based on the idea that internal common haplotypes represent ancestral haplotypes (Posada and Crandall, 2001).

An analysis of molecular variance (AMOVA, Excoffier et al., 1992) was used to investigate whether genetic variation among louse populations could be explained by patterns of variation in the host. For each species, populations were grouped based on magpie mtDNA clades such that variation was partitioned east to west. The eastern group consisted of seven populations (Philopterus sp., $n=23$; B. semiannulata, $n=42$ ) and the western group of four populations (Philopterus sp., $n=15$; $B$. semiannulata, $n=20)$ ) for each species of louse. Genetic variation was compared within all populations, among populations within groups $\left(\Phi_{\mathrm{SC}}\right)$ and between groups $\left(\Phi_{\mathrm{CT}}\right)$. $\Phi$-statistics were calculated for each level of analysis using Arlequin 3.01 (Excoffier et al., 2005).

Due to finding discordance of phylogeographic structure between host and B. semiannulata (see 'Results'), we tested COI for evidence of selection. The McDonald and Kreitman (M-K) test (Mcdonald and Kreitman, 1991) was performed in DnaSP (Rozas and Rozas, 1999). The M-K test compares the number of synonymous to nonsynonymous substitutions within and between taxa. Here we classified our taxa into two groups based on geographic distribution. The first group contained northern B. semiannulata haplotypes (clade 1). The second group contained southern haplotypes (clade 2).

\section{Results}

Genetic variation

A total of 38 Philopterus sp. individuals were sequenced for $464 \mathrm{bp}$ of COI from 11 geographic sites. The target fragment was highly variable ( 85 variable sites) and 93 mutations were found (Table 1). Twenty-three unique haplotypes were identified. No haplotype was found at more than a single geographic location. Nucleotide sequences are deposited in GenBank accession numbers EF540670-EF540693.

We identified 17 haplotypes from the $62 \mathrm{~B}$. semiannulata COI sequences. A total of 133 variable sites were found for B. semiannulata representing 133 mutations (Table 1). Twelve haplotypes were unique to individual sampling sites. The remaining five haplotypes represented $65 \%$ of all sampled individuals. Nucleotide sequences are deposited in GenBank accession numbers EF550191-EF550207.

Sequences were visually inspected for potential pseudogenes. No base ambiguities were noted between complimentary strands or within a sequence. Also there was no indication of any frameshift mutations or stop codons in either louse species sequences, suggesting all fragments sequenced were in fact from coding genes.

\section{Phylogenetics}

For the Philopterus sp. data set, maximum likelihood searches produced a single tree (Figure 2) using the model selected in Modeltest (TVM $+\mathrm{I}+\mathrm{G}, i=0.5197$; $\alpha=0.6817$ ). Five clades were strongly supported in the maximum likelihood tree reconstruction. Clade 3 was represented by only a single individual at Rowsley (Rw) and clade 5 by two individuals from the Kimberley 1 $(\mathrm{Km} 1)$ site. Clade 2 was also restricted to a single site (Alice Springs, As). Clades 1 and 4 accounted for the majority of all samples and were present at multiple sites.

A single maximum likelihood tree was produced for the $B$. semiannulata data set (Figure 3 ) using the model selected in Modeltest (GTR + G model, $i=0.0$; $\alpha=0.4529$ ). Four clades were strongly supported. Clades 3 and 4 were restricted to northeastern sites. The genetic divergence between clades 3 and 4 and all other clades was high (12.5-17.2\%). These divergent sequences were represented by only seven sampled lice and possibly reflect host transfer (see 'Discussion') and were therefore removed from further population analysis.

\section{Host-mediated genetic structure and selection}

The parsimony network analysis of the host mtDNA data set revealed two clades separated by four steps that corresponded to eastern (Cc, As, Ct, Mb, Rk, Br, Sr, Rw) and western geographical locations (Pe, Bm, Km1, Km2, Mt; Figure 4). For the Philopterus sp. data set, we were able to connect haplotypes in three of the five clades identified from the ML phylogeny. Although we were unable to connect haplotype 15 it was most closely related to clade $2(3.8 \%)$. Haplotype 20 was highly divergent from all other haplotypes (7.4-9.1\%). Philopterus sp. haplotypes were restricted to individual sites and many missing haplotypes were inferred with the network analysis. Clades 1 and 2 overlap geographically with the eastern host clade but are restricted in 
Table 1 Site locations and summary statistics for Philopterus sp. and B. semiannulata

\begin{tabular}{|c|c|c|c|c|c|c|c|}
\hline & & & $\mathrm{n}$ & hap & $S$ & $\pi$ & $\theta$ \\
\hline \multicolumn{8}{|l|}{ All populations } \\
\hline Philopterus & & & 38 & 23 & 85 & 0.05819 & 0.04360 \\
\hline Brueelia & & & 62 & 17 & 117 & 0.05639 & 0.05754 \\
\hline Alice Springs & As & $23^{\circ} 42^{\prime} \mathrm{S} 133^{\circ} 52^{\prime} \mathrm{E}$ & & & & & \\
\hline Philopterus & & & 4 & 4 & 6 & 0.00647 & 0.00705 \\
\hline Cape Crawford & $\mathrm{Cc}$ & $16^{\circ} 41^{\prime} \mathrm{S} 135^{\circ} 43^{\prime} \mathrm{E}$ & & & & & \\
\hline Philopterus & & & 1 & 1 & 0 & - & - \\
\hline Brueelia & & & 3 & 1 & 0 & - & - \\
\hline Charters Towers & $\mathrm{Ct}$ & $20^{\circ} 04^{\prime} \mathrm{S} 146^{\circ} 15^{\prime} \mathrm{E}$ & & & & & \\
\hline Philopterus & & & 5 & 2 & 11 & 0.00948 & 0.01138 \\
\hline Brueelia & & & 2 & 2 & 1 & 0.00231 & 0.00231 \\
\hline Moranbah & $\mathrm{Mb}$ & $22^{\circ} 00^{\prime} \mathrm{S} 148^{\circ} 03^{\prime} \mathrm{E}$ & & & & & \\
\hline Philopterus & & & 5 & 3 & 8 & 0.00905 & 0.00828 \\
\hline Brueelia & & & 4 & 3 & 78 & 0.09007 & 0.09826 \\
\hline Rockhampton & Rk & $23^{\circ} 22^{\prime} \mathrm{S} 150^{\circ} 30^{\prime} \mathrm{E}$ & & & & & \\
\hline Philopterus & & & 1 & 1 & 0 & - & - \\
\hline Brueelia & & & 8 & 2 & 58 & 0.03349 & 0.05166 \\
\hline Brisbane & $\mathrm{Br}$ & $27^{\circ} 28^{\prime} \mathrm{S} 153^{\circ} 01^{\prime} \mathrm{E}$ & & & & & \\
\hline Brueelia & & & 8 & 6 & 104 & 0.11358 & 0.09263 \\
\hline Seymour & $\mathrm{Sr}$ & $37^{\circ} 01^{\prime} \mathrm{S} 145^{\circ} 09^{\prime} \mathrm{E}$ & & & & & \\
\hline Philopterus & & & 6 & 3 & 9 & 0.00920 & 0.00849 \\
\hline Brueelia & & & 15 & 6 & 5 & 0.00326 & 0.00355 \\
\hline Rowsely & $\mathrm{Rw}$ & $37^{\circ} 43^{\prime} \mathrm{S} 144^{\circ} 24^{\prime} \mathrm{E}$ & & & & & \\
\hline Philopterus & & & 1 & 1 & 0 & - & - \\
\hline Brueelia & & & 2 & 1 & 0 & - & - \\
\hline Perth & $\mathrm{Pe}$ & $31^{\circ} 53^{\prime} \mathrm{S} 115^{\circ} 51^{\prime} \mathrm{E}$ & & & & & \\
\hline Brueelia & & & 10 & 2 & 1 & 0.00123 & 0.00082 \\
\hline Broome & $\mathrm{Bm}$ & $17^{\circ} 57^{\prime} \mathrm{S} 122^{\circ} 14^{\prime} \mathrm{E}$ & & & & & \\
\hline Philopterus & & & 1 & 1 & 0 & - & - \\
\hline Brueelia & & & 3 & 1 & 0 & - & - \\
\hline Kimberley1 & $\mathrm{Km} 1$ & $16^{\circ} 49^{\prime} \mathrm{S} 124^{\circ} 55^{\prime} \mathrm{E}$ & & & & & \\
\hline Philopterus & & & 7 & 4 & 44 & 0.04495 & 0.03871 \\
\hline Brueelia & & & 3 & 2 & 1 & 0.00154 & 0.00154 \\
\hline Kimberley2 & $\mathrm{Km} 2$ & $15^{\circ} 42^{\prime} \mathrm{S} 126^{\circ} 22^{\prime} \mathrm{E}$ & & & & & \\
\hline Philopterus & & & 4 & 2 & 3 & 0.00431 & 0.00353 \\
\hline Mataranka & Mt & $14^{\circ} 55^{\prime} \mathrm{S} 133^{\circ} 04^{\prime} \mathrm{E}$ & & & & & \\
\hline Philopterus & & & 3 & 1 & 0 & - & - \\
\hline Brueelia & & & 4 & 3 & 78 & 0.09007 & 0.09826 \\
\hline
\end{tabular}

$n$, number of individual lice typed for each locality. Only one louse per bird was included in the analysis. hap, number of haplotypes present; $\mathrm{S}$, segregating sites; $\pi$, nucleotide diversity.

distribution. Clade 1 was only found at the As site whereas clade 2 had a broader distribution and was found at all other eastern sites $(\mathrm{Ct}, \mathrm{Mb}, \mathrm{Rk}, \mathrm{Br}, \mathrm{Sr}, \mathrm{Rw})$ except As and Cape Crawford (Cc). Clade 3 overlapped with the host's western clade, however one haplotype was found on eastern magpies at Cc.

The geographical distribution of $B$. semiannulata mtDNA haplotypes produced a very different pattern to that of both Philopterus sp. and the Australian magpie. Two non-overlapping clades, clades 1 (southern Australia) and 2 (northern Australia) accounted for the majority of haplotypes and represented the entire sampling distribution. Divergence between the northern and southern clades was $4.2 \%$. The ancestral haplotype (Figure 4) inferred for clade 2 was found at all northern sites (Bm, Km1, Km2, Mt, Cc, Ct, Mb, Rk). The ancestral haplotype inferred for clade 1 was found in southeastern sites (Br, Sr, Rw), whereas the southwestern site Perth (Pe) was represented by two unique haplotypes that were separated by two steps from southeastern haplotypes.

Based on host mtDNA groups, the AMOVA indicated a high level of among population within group variation in Philopterus sp. $\left(\Phi_{\mathrm{SC}}=0.74, P<0.001\right)$ and B. semiannu- lata $\left(\Phi_{\mathrm{SC}}=0.95, P<0.001\right)$. The between-group analysis revealed however, remarkably different results for each species of louse. For the Philopterus sp. data set, $74.6 \%$ of the variation was partitioned between eastern and western groups. However, the groups did not account for any of the variation $(-37.2 \%)$ in B. semiannulata sequence data. The $\Phi_{\mathrm{CT}}$ was highly significantly for Philopterus sp. $\left(\Phi_{\mathrm{CT}}=0.75, P<0.05\right)$, yet was not significant in B. semiannulata $\left(\Phi_{\mathrm{CT}}=-0.37, P=0.89\right)$.

The $\mathrm{M}-\mathrm{K}$ test was not significant $(P=0.53)$ suggesting there was no departure from neutrality for the COI gene of $B$. semiannulata. In fact, there were very few nonsynonymous substitutions (fixed $=1$, polymorphic $=2$ ) compared to synonymous substitutions (fixed $=18$, polymorphic $=8$ ) between the two clades.

\section{Discussion}

Population structure of feather lice

As feather lice are obligate parasites and are unable to survive for long away from their host, we expected to see a concordant pattern of variation between lice and their host. However, in contrast to what we expected, 


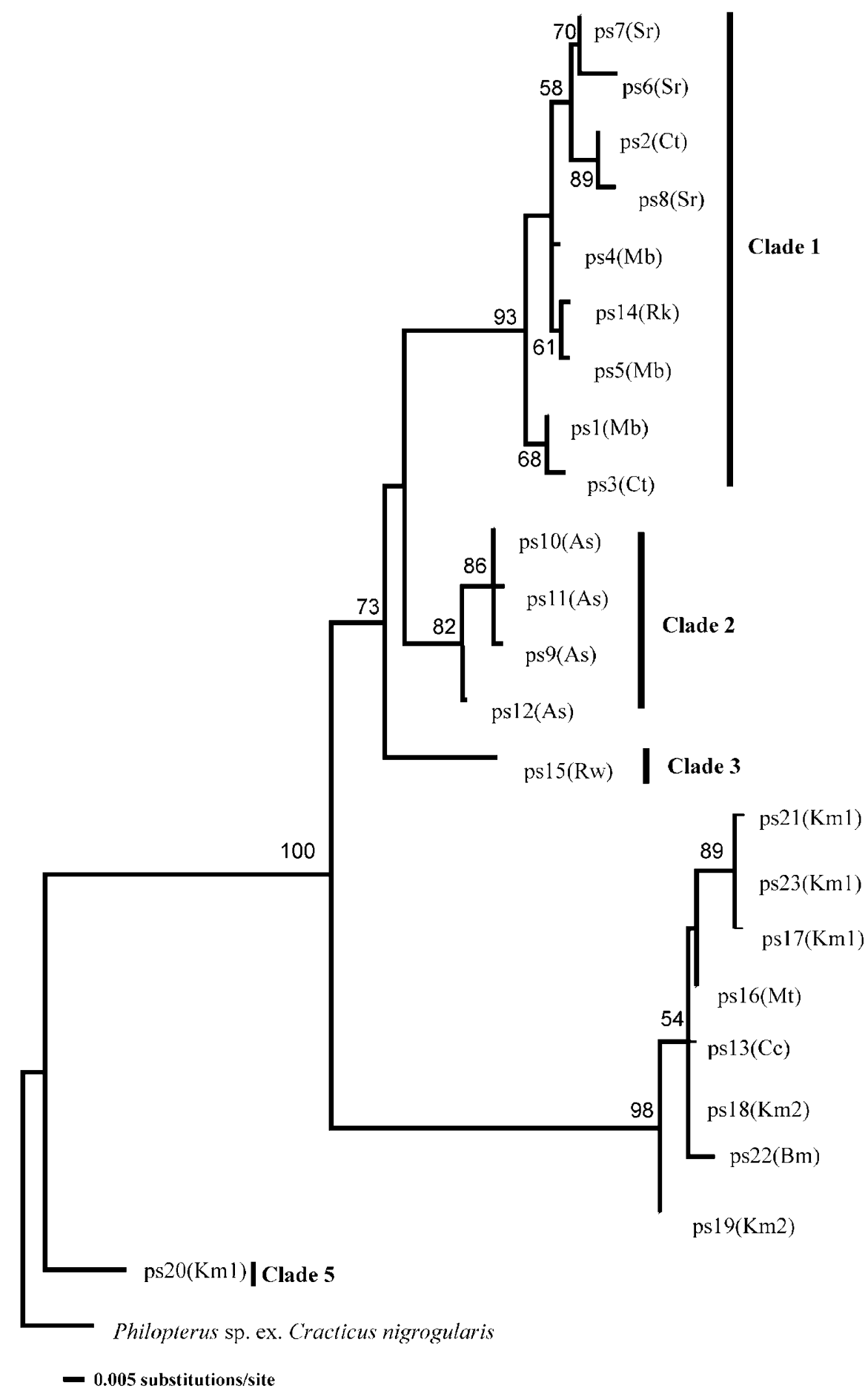

Clade 4

Figure 2 Phylogram estimated with maximum likelihood analysis for Philopterus sp. based on a fragment of cytochrome oxidase I (COI) mitochondrial gene. Topology was rooted with Philopterus sp. ex. C. nigrogularis. Numbers on branch nodes indicate $>50 \%$ support for 100 bootstrap replicates.

phylogenetic analysis of two species of magpie feather lice showed two distinctly different patterns of geographic variation. Philopterus sp. showed deep east-west divergence congruent with phylogeographic structure shown in the host. B. semiannulata showed phylogeographic structure, yet was discordant with the magpie host. Several B. semiannulata and two Philopterus sp. individuals that were sequenced were highly divergent to all other samples and were in the range observed between species in the louse genus Physconelloides (Johnson et al., 2002) or among species of Columbicola that use different hosts (Whiteman et al., 2004). These lice possibly represent host transfer from their regular host to the Australian magpie.

At the broad scale, phylogeographic structure of Philopterus sp. was congruent with the host, both 


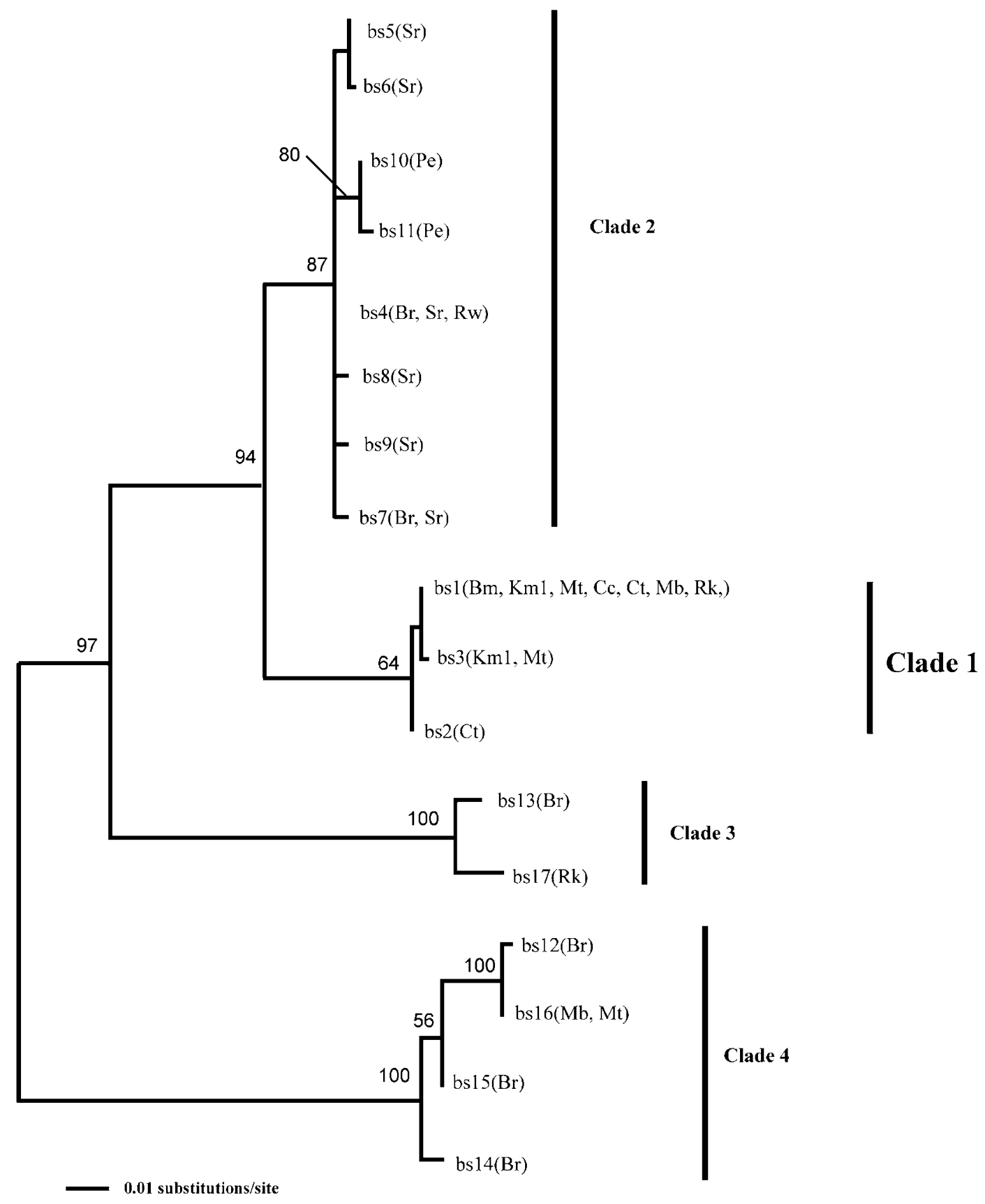

Figure 3 Phylogram estimated with maximum likelihood analysis for B. semiannulata based on a fragment of cytochrome oxidase I (COI) mitochondrial gene. Topology was midpoint rooted. Numbers on branch nodes indicate $>50 \%$ support for 100 bootstrap replicates.

displaying deep east-west divergence. No haplotypes were shared between eastern and western populations of Philopterus sp. and the AMOVA based on magpie mtDNA clades estimated a very large, highly significant $\Phi_{\mathrm{CT}}(0.75, P<0.05)$. The position of the break between eastern and western lineages in Philopterus sp. differed slightly from the magpie host. Philopterus sp. collected from Cape Crawford (Cc) belonged to a mtDNA clade restricted to northwestern populations, whereas magpies from the same site belonged to the eastern mtDNA magpie clade (Toon et al., 2007). Although no secondary contact was suggested between host mtDNA clades, gene flow was inferred from microsatellite data between northeastern and northwestern populations thereby potentially facilitating dispersal of western lice to eastern magpie hosts.

Population genetic structure is greater in Philopterus sp. than in the host and thus phylogeographic patterns in this species of louse may provide an insight into very recent history of magpies. Apart from the eastern and western haplotypes found in roughly the same geographic distribution as magpie clades, a third clade was found at the As site. Mitochondrial and microsatellite analysis of magpie populations suggests that Alice Springs (As) is not differentiated from other eastern sites (Toon et al., 2007). The presence of a divergent clade of 
a
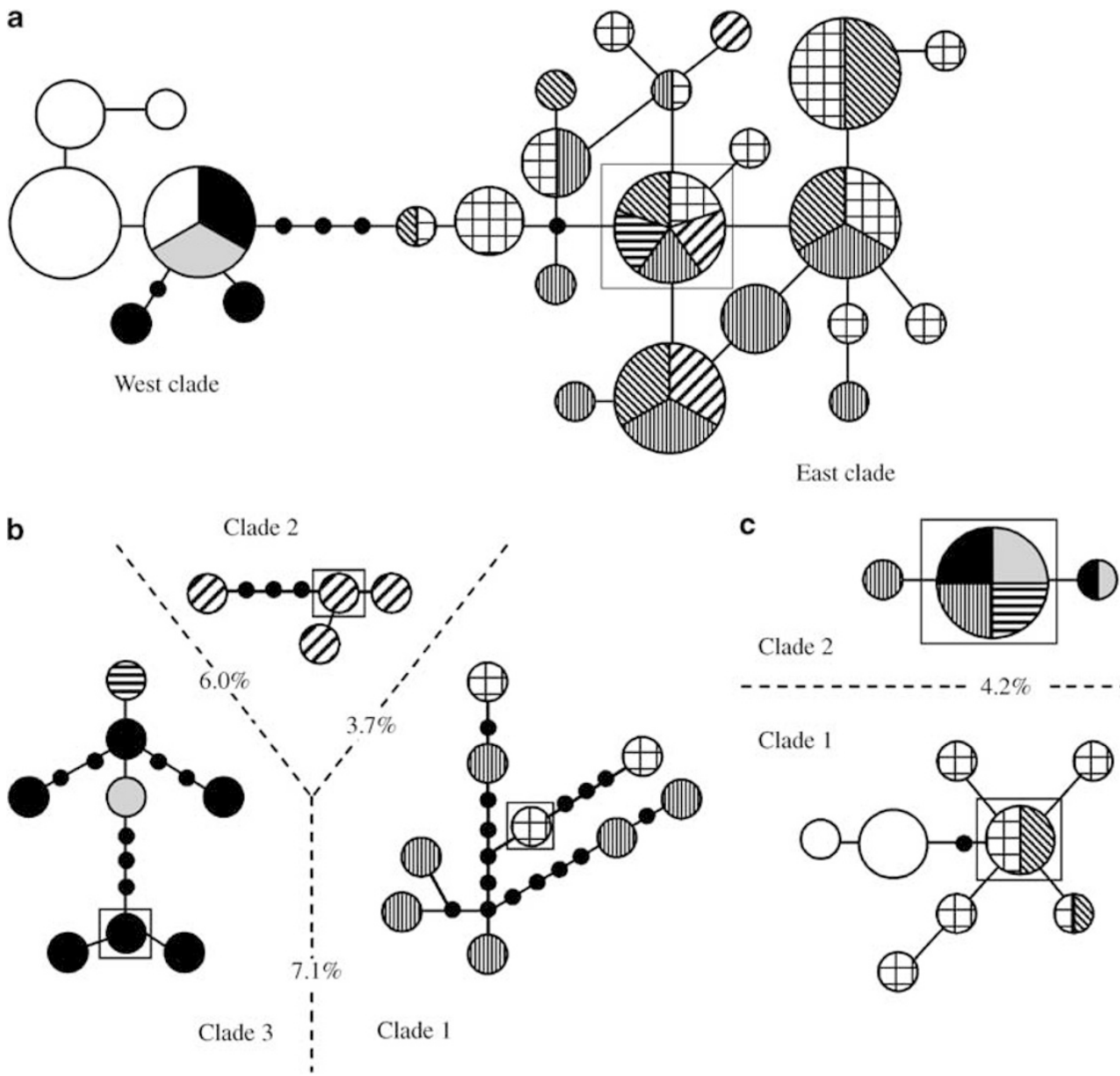

Clade 2

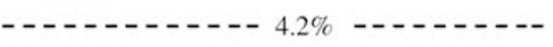

Clade 1

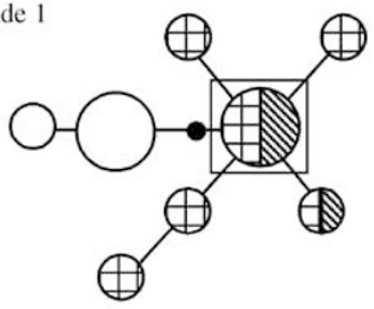

Figure 4 Parsimony networks computed by TCS using mtDNA sequence data of (a) G. tibicen, (b) Philopterus sp. and (c) B. semiannulata. Geographical location of sampled haplotypes is indicated by haplotype patterns, which correspond to Figure 1. Size of circle represents number of sampled individuals: small circles indicate less than 5 individuals, medium circles are 5-20 individuals and large circles are more than 20 individuals sampled. Boxes around haplotypes indicate the oldest inferred haplotype following Castelloe and Templeton (1994).

Philopterus sp. at the As site but not for magpies may suggest that this population has been isolated historically for a short period of time but not for sufficient time to show divergence in the host. It is postulated that during glacial/arid periods in the Pleistocene, Australia was divided by arid barriers into at least six coastal refuges and an inland refuge in central Australia (Keast, 1961). From mtDNA analysis and estimates of population divergence, it is suggested that magpies were divided into at least two populations by the time of the most recent glacial/arid period (100000-16000 BP) that were most likely distributed along the east coast and the south west of the continent (Toon et al., 2007). It is possible that magpies also inhabited a third refuge in central Australia during the last glacial/arid period for a short period of time that was sufficient to allow divergence in the parasite and not in the host. An alternative explanation is that dispersal of magpies among eastern populations may not necessarily facilitate dispersal of Philopterus sp. over large distances. Dispersal of Philopterus sp. requires either vertical transmission via direct host to host contact (within families/territories) or horizontal transmission on other hosts (for example, phoresis on hippoboscid flies) (Keirans, 1975). If transmission of Philopterus sp. is less than the dispersal of magpies we would expect the parasite to show greater genetic structure than the host. Among the eastern Philopterus sp. populations there appears to be considerable divergence (within divergence $=0.7-1.6 \%$ ) suggesting that transmission of Philopterus sp. may be restricted. To test if the As population is divergent due to dispersal or vicariance, population analysis comparing gene flow over a small versus a large scale to estimate dispersal potential of Philopterus sp. is required.

In contrast to the concordance of phylogeographic structure between Philopterus sp. and the host, B. semiannulata showed a non-overlapping north-south distribution of divergent clades. Louse haplotypes were shared between east and west populations of the host and the AMOVA based on host mtDNA clades was not significant. One common haplotype (bs1) within the northern clade (clade 2) was found in all northern populations distributed from the Kimberley $(\mathrm{Km})$ region across to Rockhampton (Rk) in eastern Australia over a distance greater than $3000 \mathrm{~km}$. Although maternal gene flow is absent in the host between northeast and northwest Australia, microsatellite data suggested that male magpie dispersal is occurring between northeast and northwest Australia (Toon et al., 2007). It is possible that $B$. semiannulata disperse primarily on male magpies 
across northern Australia. Dispersal across northern Australia may also be facilitated via phoresis, the horizontal transfer of lice to other hosts on hippoboscid flies (Keirans, 1975). During the course of this study hippoboscid flies were commonly observed on captured wild magpies. Brueelia spp. have also been observed on hippoboscid flies collected from dead magpies (Hughes, 1980). Although dispersal of B. semiannulata across northern Australia may be explained by male magpiemediated dispersal or phoresis, the question remains why there is a large population divergence $(4.2 \%)$ observed between northern and southern populations where there is no host barrier to gene flow?

Similar phylogeographic patterns between louse and host were expected based on the assumption of shared history. However, this idea assumes vertical transmission of the parasite and sharing of ecological constraints between parasite and host. Empirical studies have shown that dispersal ability (Clayton and Johnson, 2003; Whiteman et al., 2007) of lice underlies patterns of population structure. In addition, ecological constraints such as humidity (Carrillo et al., 2007) and size of host (Clayton et al., 2003) may restrict the distribution of a species of louse. Although B. semiannulata structure was not congruent with the host, louse clades were restricted to either northern or southern magpie populations suggesting there is some geographical association. This association may be due to different geographic lineages dispersing via different species of hippoboscid flies with disjunct geographical distributions. Alternatively, populations of $B$. semiannulata may be restricted in distribution due to habitat constraints. B. semiannulata lives on body feathers of the Australian magpie that vary in colour and size. Colour of back feathers changes abruptly (approximately $100 \mathrm{~km}$ in the south east) between northern and southern populations and this morphological feature is used to classify magpies into three main back colour forms: northern black-backs, southeastern white-backs and southwestern varied (Schodde and Mason, 1999). These back colour forms are however, not strictly associated with $B$. semiannulata lineages. Southern B. semiannulata populations are found at Brisbane $(\mathrm{Br})$ over $1000 \mathrm{~km}$ north of the contact zone (Figure 1) between black-backs and white-backs in eastern Australia. Therefore, even if lice diverged due to differences in back colour of host it is unlikely these differences are currently maintaining the geographic distribution of lineages.

Size of magpie also changes latitudinally, and represents a cline such that southeastern magpies are the largest (wing: males 230-255 $\mathrm{mm}$ ) and northern magpies (wing: males 270-290 mm) are the smallest mainland birds (Schodde and Mason, 1999). Clayton et al. (2003) demonstrated a relationship between host body size (proportional to feather size) and lice body size and found that this relationship was important for lice to avoid being removed from their host during preening. The distribution of divergent $B$. semiannulata lineages may reflect taxa that are restricted by feather size of the magpie host. Experimental analysis of feather size and behaviour of $B$. semiannulata in relation to preening is required to test this hypothesis.

In conclusion, the two species of Australian magpie feather lice examined here showed very different phylogeographic patterns. Philopterus sp. are strongly congruent with their magpie host reflecting a shared history and supporting the idea of vertical transmission as a means of dispersal. In contrast, phylogeographic patterns in B. semiannulata are much more complex and are possibly influenced by dispersal of the louse and ecology of the host rather than reflecting a shared history with the host.

\section{Acknowledgements}

We thank Andrew Baker, Giovannella Carini, Corinna Lange, Kate Durrant, James Fawcett, Joel Huey, Steve Smith, Jemma Sommerville, Kate Masci, Dan Schmidt, Adele Schmidt, Tim Page and Kaye Stuart for their fantastic assistance in the field. We also thank Peter Mather for suggestions during this project and two anonymous reviewers for their helpful comments on the article. Thank you to Ricardo Palma for valuable taxonomic advice. Samples were collected under Western Australian Department of Conservation and Land Management permit SF003903, Queensland Parks and Wildlife permit W4/002670/01/SAA, Northern Territory Parks and Wildlife Commission permit 18145 and Victorian Wildlife permit 10003058.

\section{References}

Baker AM, Mather PB, Hughes JM (2000). Population genetic structure of Australian magpies: evidence for regional differences in juvenile dispersal behaviour. Heredity 85: 167-176.

Bensasson D, Zhang D-X, Hartl D, GM H (2001). Mitochondrial pseudogenes: evolution's misplaced witnesses. Trends Ecol Evol 16: 314-321.

Carrillo CM, Valera F, Barbosa A, Moreno E (2007). Thriving in an arid environment: high prevalence of avian lice in low humidity conditions. Ecoscience 14: 241-249.

Castelloe J, Templeton AR (1994). Root probabilities for intraspecific gene trees under neutral coalescent theory. Mol Phylogenet Evol 3: 102-113.

Clay T (1951). An introduction to a classification of the avian Ischnocera (Mallophaga): part I. Trans $R$ Ent Soc Lond 102: 171-194.

Clayton DH, Bush SE, Goates BM, Johnson KP (2003). Host defense reinforces host-parasite cospeciation. Proc Natl Acad Sci 100: 15694-15699.

Clayton DH, Johnson KP (2003). Linking coevolutionary history to ecological process: doves and lice. Evolution 57: 2335-2342.

Clement M, Posada D, Crandall KA (2000). TCS: a computer program to estimate gene genealogies. Mol Ecol 9: 1657-1659.

Covacin C, Shao R, Cameron S, Barker SC (2006). Extraordinary number of gene rearrangements in the mitochondrial genomes of lice (Phthiraptera: Insecta). Insect Mol Biol 15: 63-68.

Criscione CD, Blouin MS (2007). Parasite phylogeographical congruence with salmon host evolutionarily significant units: implications for salmon conservation. Mol Ecol 16: 993-1005.

Criscione CD, Cooper B, Blouin MS (2006). Parasite genotypes identify source populations of migratory fish more accurately than fish genotypes. Ecology 87: 823-828.

Dybdahl MF, Lively CM (1996). The geography of coevolution: comparative population structures for a snail and its trematode parasite. Evolution 50: 2264-2275.

Excoffier L, Laval F, Schneider S (2005). Arlequin ver 3.0: an integrated software package for population genetics data analysis. Evol Bioinf Online 1: 47-50.

Excoffier L, Smouse PE, Quattro JM (1992). Analysis of molecular variance inferred from metric distances among DNA haplotypes: application to human mitochondrial DNA restriction data. Genetics 131: 479-491. 
Folmer O, Black M, Hoeh W, Lutz R, Vrijenhoek R (1994). DNA primers for amplification of mitochondrial cytochrome $c$ oxidase subunit I from diverse metazoan invertebrates. Mol Mar Biol Biotechnol 3: 294-299.

Heilveil JS, Berlocher SH (2006). Phylogeography of postglacial range expansion in Nigronia serricornis Say (Megaloptera: Corydalidae). Mol Ecol 15: 1627-1641.

Hughes JM (1980). Geographic Variation in the Australian Magpie and its Mallophagan Parasites. La Trobe University: Melbourne.

Hughes JM (1984a). Distribution of Mallophaga on the Australian magpie (Gymnorhina tibicen Latham) (Family: Cracticidae). Aust Zool 21: 459-465.

Hughes JM (1984b). Morphometric variation in the Mallophaga of the Australian magpie (Gymnorhina tibicen Latham). Aust Zool 21: 467-477.

Johnson KP, Williams BL, Drown DM, Adams RJ, Clayton DH (2002). The population genetics of host specificity: genetic differentiation in dove lice (Insecta: Phthiraptera). Mol Ecol 11: $25-38$.

Keast A (1961). Bird speciation on the Australian continent. Bull Mus Comp Zool 123: 307-495.

Keirans J (1975). A review of the phoretic relationship between Mallophaga (Phthiraptera: Insecta) and Hippoboscidae (Diptera: Insecta). J Med Entomol 12: 71-76.

Marshall AG (1981). The Ecology of Ectoparasite Insects. Academic Press: London.

McCoy KD, Chapuis E, Tirard C, Boulinier T, Michalakis Y, Le Bohec C et al. (2005). Recurrent evolution of host-specialized races in a globally distributed parasite. Proc $R$ Soc B Biol Sci 272: 2389-2395.

McDonald JH, Kreitman M (1991). Adaptive protein evolution at the Adh locus in Drosophila. Nature 351: 652-654.

Nadler SA (1995). Microevolution and the genetic structure of parasite populations. J Parasitol 81: 395-403.

Nei M, Li W-H (1979). Mathematical model for studying genetic variation in terms of restriction endonucleases. Proc Natl Acad Sci 76: 5269-5273.
Nieberding C, Morand S, Libois R, Michaux JR (2004). A parasite reveals cryptic phylogeographic history of its host. Proc R Soc Lond Ser B Biol Sci 271: 2559-2568.

Nieberding CM, Olivieri I (2007). Parasites: proxies for host genealogy and ecology? Trends Ecol Evol 22: 156-165.

Page RDM, Lee PLM, Becher AA, Griffiths R, Clayton DH (1998). A different tempo of mitochondrial DNA evolution in birds and their parasitic lice. Mol Phylogenet Evol 9: 276-293.

Posada D, Crandall KA (1998). MODELTEST: testing the model of DNA substitution. Bioinformatics 14: 817-818.

Posada D, Crandall KA (2001). Intraspecific gene genealogies: trees grafting into networks. Trends Ecol Evol 16: 37-45.

Price RD, Hellenthal RA, Palma RL, Johnson KP, Clayton DH (2003). The Chewing Lice: World Checklist and Biological Overview. Natural History Survey: Illinois.

Rozas J, Rozas R (1999). DnaSP version 3: an integrated program for molecular population genetics and molecular evolution analysis. Bioinformatics 15: 174-175.

Schodde R, Mason IJ (1999). The Directory of Australian Birds: Passerines. CSIRO Publishing: Melbourne.

Swofford D (2000). PAUP: Phylogenetic Analysis Using Pasrsimony, Version 4.0b4a. Sinauer: Sunderland, MA.

Toon A, Mather PB, Baker AM, Durrant KL, Hughes JM (2007). Pleistocene refugia in an arid landscape: analysis of a widely distributed Australian passerine. Mol Ecol 16: 2525-2541.

Whiteman NK, Kimball RT, Parker PG (2007). Co-phylogeography and comparative population genetics of the threatened Galápagos hawk and three ectoparasite species: ecology shapes population histories within parasite communities. Mol Ecol 16: 4759-4773.

Whiteman NK, Sanchez P, Merkel J, Klompen H, Parker PG (2006). Cryptic host specificity of an avian skin mite (Epidermoptidae) vectored by louseflies (Hippoboscidae) associated with two endemic Galapagos bird species. J Parasitol 92: 1218-1228.

Whiteman NK, Santiago-Alarcon D, Johnson KP, Parker PG (2004). Differences in straggling rates between two genera of dove lice (Insecta: Phthiraptera) reinforce population genetic and cophylogenetic patterns. Int J Parasitol 34: 1113-1119. 